\title{
Services Supporting Knowledge Maturing in Small and Medium-Sized Enterprises
}

\author{
Ronald Maier \\ School of Management, Information Systems \\ University of Innsbruck, Austria
}

\begin{abstract}
The hype around Web 2.0 has again sparked tremendous interest in IT-supported knowledge management and technology-enhanced learning in organizations. Although there has been abundant evidence of how to benefit from Web 2.0 technologies, information on how to go about deploying these in small and medium-sized enterprises in a coordinated manner are scarce. Based on the findings of an empirical study and an ethnographically informed study on knowledge maturing, this paper suggests a set of knowledge services to support a series of knowledge actions chained with the help of the knowledge maturing model. This set of services can be used by small and medium-sized enterprises for analyzing IT tools and systems which currently support their employees' knowledge maturing activities, to foster knowledge cooperation with customers, suppliers and partners in their business environment and to find gaps which can be filled particularly by consuming services over the Web.
\end{abstract}

\section{Introduction}

Work in organizations is increasingly information- and knowledge-intensive and the share of knowledge work has risen continuously during the last decades (Wolff, 2005). Since the late 90s, organizations have been faced with the transformation to knowledge-intensive organizations in order to significantly increase speed of innovation and improve productivity of knowledge work (Drucker, 1994). Knowledgeintensive organizations represent a substantial share of small and medium-sized enterprises (SMEs) which are considered the backbone of innovation in the European economy. Knowledge intensity refers to, among other things, a high share of highly skilled and/or creative employees, operations that aim at providing knowledgeintensive products and services, high importance of experiences, high degree of innovations, in some industry sectors this results in a high number of patents, central importance of customer knowledge, high need for communication and a high degree of information needs (Starbuck 1992, Alvesson 2004). However, compared to more traditional, predominantly manual, data- or service-oriented work, the unstructured, creative and expertise-driven knowledge work cannot be designed with standardized management approaches and cannot be easily supported by information and communication technologies (ICT), e.g., workflows or single application systems.

Resorting to Knowledge management (KM) has been suggested to help solve these issues. During the last twenty years, businesses have faced four distinctive phases of 
KM. The first phase could be termed human-oriented KM. Organizations realized the value of their "human capital" and bundled a number of instruments aiming at the individual knowledge worker and her productivity. The next phase was backed by tremendously increased opportunities offered by ICTs and could be called technologyoriented KM. Organizations were eagerly experimenting with new ICTs in attempts to benefit from the promised changes that would come about by implementing KM tools and systems. In a third phase which primarily was fueled by the emphasis on business processes typical for German-speaking countries, KM methods, tools and instruments were repositioned as knowledge processes and linked to knowledge-intensive business processes. Thus, KM initiatives could be designed with the same language as was used in organizational design and ICT support of business activities in general, the language of business processes. After human-oriented, technology-oriented and process-oriented $\mathrm{KM}$, recently a fourth KM phase has reached businesses backed by the hype keywords Web 2.0 and social software: collaborative KM. While in many organizations knowledge workers are busy trying out new alternatives for production of contents, for networking and for self-directed learning, questions arise how these activities can be coordinated or guided so that they are in line with organizational goals.

As a result, an enormous number of fragmented KM measures, procedures, instruments and tools have been proposed which claim to solve particular knowledgerelated problems, but are not connected or integrated. Even though many authors have studied the strategic perspective of KM, e.g., (April, 2002; Hansen et al. 1999; Ordóñez de Pablos, 2002; Zack, 1999), process-oriented KM strategies in particular (Davenport et al. 1996), in order to integrate KM initiatives and guide their organization-wide implementation, these considerations still remain on an abstract level. Particularly actors designing KM initiatives for SMEs are overwhelmed by the number of measures, procedures, instruments and tools proposed in the literature and thus require guidance in selecting and composing services to support those knowledge activities that are deemed most valuable in their business context.

This paper argues that composition or integration of knowledge services in organizations requires their alignment with the help of the knowledge maturing model (Maier \& Schmidt 2007) that connects them according to a set of knowledge activities. Section 2 briefly introduces the concept of knowledge service. Section 3 describes the knowledge maturing model. Section 4 presents a list of knowledge services supporting the activities defined in the knowledge maturing model. Section 5 finally discusses application of the model in KM initiatives for SMEs and concludes the paper.

\section{Knowledge Services}

Since software engineering was founded as a discipline in the 70s, computer scientists have searched for ways to describe basic or advanced building blocks out of which software systems can be composed. The main advantages are reduced cost and time as well as improved quality through modularization, reusability, stability and interoperability of the resulting software systems. There are a number of different terms for building blocks, e.g., functions, procedures, modules, classes or components. Recently, service has been the central concept for a redefinition of the technical and conceptual 
foundation for these main building blocks from a more business-oriented perspective. A service consists of contract, interface and implementation. It has distinctive functional meaning typically reflecting a high-level business concept covering data and business logic (Krafzig et al. 2005). A service is an abstract resource that represents a capability of performing tasks that form a coherent functionality from the point of view of the providers entities and requesters entities (W3C 2004). Service descriptions provide information about:

- service capability: conceptual purpose and expected result,

- service interface: the service's signature, i.e. input, output, error parameters and message types,

- service behavior: a detailed workflow invoking other services,

- quality of service: functional and non-functional quality attributes, e.g., service metering, costs, performance metrics and security attributes.

The service concept has gained popularity with the advent of a set of standards (i.e. URI, XML, UDDI, SOAP and WSDL) for open interaction between software applications using Web services. Web services are one way of implementing business and technical services in a service-oriented architecture (SOA). A SOA comprises application frontend, services, service repository and service bus which make functions available so that they can be accessed without any information about their implementation. SOAs promise more flexibility and adaptability In the context of SOA, services have to be interoperable in terms of platform independence, own a network addressable interface and be dynamically discovered and accessed. "SOA-enabled" organizations are called agile, on-demand or service-oriented enterprises. These metaphors attempt to carry over SOA semantics to organizational design. This has connotations for changes in ICT's general role in business (transforming business models), value creation (value networks), business processes (dynamically designed, net-like with emphasis on parallel processing) as well as organizational structure (service consumer-provider relationship complementing or even replacing traditional hierarchies). Whereas the technical definition of services is supported by a set of standards, it is the conceptual part (i.e. defining types of services that are useful) that is currently lacking.

Knowledge services are a subset of services whose functionality supports highlevel KM instruments as part of on-demand KM initiatives, e.g., find expert, submit experience, publish skill profile, revisit learning resource or join community-ofinterest. For example, a knowledge service "search for experts" might be composed of the basic services (1) expert search, (2) keyword search, (3) author search, (4) employee search and (5) check availability. The (1) expert search service delivers a list of IDs, e.g., personnel numbers, for experts matching the input parameter of an area of expertise. The (3) author search service requires a list of keywords describing the area of expertise provided by a (2) keyword search service. Keywords are assigned to areas of expertise in a database solution or in a more advanced semantic integration system based on ontology. With the help of an inference engine, these relationships, together with rules in the ontology, can be used to determine a list of keywords. The (3) author search service then returns a list of IDs of matching authors or active contributors to the CMS. An (4) employee search service takes the personnel numbers found in expert and author search and returns contact details, e.g., telephone number, 
email address, instant messaging address. Finally, the (5) check availability service delivers the current status of the experts and a decision on their availability. Knowledge services describe a set of services provided by heterogeneous application systems that can be arranged to support activities of knowledge maturing.

However, SOA and services are concepts that so far mostly impact large organizations, because creating a SOA is a costly and complex undertaking. Furthermore, it remains difficult to show that the value of KM initiatives exceeds corresponding efforts. Also, KM tools often need a "critical mass" of contributors which is much easier to achieve in large organizations than in SMEs. Therefore, many KM projects, particularly in SMEs, have been abandoned leaving knowledge workers with the insight that KM is important, yet left unsupported. The developments termed as Web 2.0 provide the "raw material" for a solution to these challenges as they offer cheap, easy-to-use technologies that are used by a broad range of people from which SMEs can profit. As Web 2.0 is a hype term rarely defined, in the following lines, some characteristics differentiating Web 2.0 from its predecessor are discussed from the perspective of how they can be beneficial for SMEs (after O'Reilly 2005).

Web as platform: Software is developed not to work in the environment of a single vendor's operating system, but on the Web tied together by a set of open interaction standards that are the result of agreements between major players in Web development and usage. This gives rise to benefits for SMEs as they can avoid vendor lock-in effects as well as profit from the benefits of combining Web services offered by different sites to present them to employees.

Network effects: Harnessing collective intelligence means services get more value with every increase of the number of people using it. Important phenomena typically related to goods and services that follow a pattern of network effects are start-up problems, switching costs and lock-in effects. The start-up problem describes the effect related to the low benefits of the service right after its start with nobody (yet) using it, it is difficult to promote a new service. Switching costs mean the effect that switching would require all knots with which one is connected to also switch to a new service thus creating lock-in effects. Due to small numbers of active participants in SMEs, these effects are of particular importance as they might prevent application of potentially useful tools. This calls for providing user-generated content and services in a form that allows remixing by others in the business ecosystem of the SME or even beyond and thus sparks an "architecture of participation" crossing organizational boundaries.

Value of data: User-generated content is the single most important asset in typical Web 2.0 applications. Several start-up companies have quickly made a fortune by attracting large numbers of users to provide content. Some Web 2.0 applications also consume and remix data from multiple sources. Strictly speaking, a plethora of data without users coming back to the site is worth nothing. So, it is data plus (returning) people that drive a Web site. SMEs can profit on the one hand from the plethora of data that can be remixed and consumed by them and on the other hand open up portions of their data to have other people help check it and improve its quality.

Webtop instead of Desktop: Interaction with the Web used to be strongly limited when compared to a Desktop application. With the advent of XHTML, CSS, DOM, XMLHttpRequest and Javascript, bundled under the term of AJAX, it has become possible to create Web sites that allow for rich user experiences, i.e. a look-and-feel 
similar to Desktop applications. Thus, Web 2.0 applications are sufficiently user friendly to be applied even by those SME employees who are not very technologysavvy.

End of the software release cycle: Software in the Web 2.0 is continuously developed and consequently in a permanent Beta status. Software is therefore delivered as a continually-updated service with no versioning or releases. SMEs can benefit by allowing them to realize quick wins even before large investments need to be taken and have services co-tested and developed by people within and outside the SME so that they gradually and quickly get better.

Software above the level of a single device: Many people do not only have a PC at their disposal, but also other information devices e.g., laptops, personal digital assistants or smartphones which share the ability to connect to the Web. One aspect of Web 2.0 is also accessibility of contents and services from varying types of devices. The single most important factor is that many people tend to have mobile devices with them almost all of the time, which gives rise to benefits also in those SMEs in which most employees do not continuously work on a PC.

Lightweight models: Web 2.0 stands for the opposite of heavyweight programming models typically used for application design in intra-organizational settings of large organizations. Web 2.0 applications are loosely coupled systems, that are designed for remixability and their innovation is in assembly, not in creating new entire systems in an isolated way. SMEs with their often higher flexibility compared to large organizations can potentially quickly move to these models and profit from first-mover advantages.

To sum up, with more light-weight Web 2.0 technologies such as tagging, RSS and mash-ups and the corresponding network effects created by numerous people using contents and services offered partly freely over the Web, also individuals with their personal knowledge environments and consequently SMEs comprising individuals with their personal work environments can benefit from arranging services flexibly to help them fulfill their knowledge needs. The personal, collaborative KM initiatives, often associated with haphazard, trial-and-error, grass-roots level approaches, need to be guided without losing the momentum created in KM activities by individuals. This is the main goal of the knowledge maturing model presented in the following section.

\section{Knowledge Maturing}

As described above, numerous systems aim at improving knowledge and learning processes which are typically designed and managed according to the specific needs of the respective organization. Employees thus use a fragmented systems landscape in which each system supports a certain part of knowledge and learning processes. Specific to knowledge-intensive SMEs is the fact that often many knowledge processes cross organizational boundaries, and employees use a considerable number of tools and systems provided by other institutions, mostly over the Web. There are substantial conceptual challenges of designing learning and knowledge processes that bring together the separated organizational support infrastructures fostered by different organizations. Organizations and corresponding application systems typically target knowledge of different degrees of maturity. 
Pruning the tree of types of knowledge elements and guiding employees on how to use the channels of knowledge transfer is a pivotal task in any KM initiative. In the following paras, the knowledge maturing process is described in order to provide a framework for designing and integrating types of knowledge elements, processes and channels in KM. In a first step of structuring this process, Figure 1 shows the five phases that have been identified after analyzing several practical cases in applied research projects (Schmidt 2005), on the basis of a large empirical study (Maier 2007) as well as an ethnographically informed study on individual knowledge maturing routines (Maier \& Thalmann 2008). Figure 1 lists a number of key concepts that explain the individual steps of the knowledge maturing model. The steps are further differentiated with the help of:

- Drives: Evolution theory postulates that during evolution humans have developed a set of innate psychological mechanisms that drive their behavior: acquire, defend, bond, comprehend (after Watson 2008) and pass-on.

- Motives: There are numerous motivation theories explaining human behavior as an aim to satisfy a set of motives (many of which build on Maslow 1943). Motives that drive the knowledge maturing process are social belonging, power, status, curiosity and self-realization.

- Actions: The steps typically involve many (knowledge) actions, e.g., access, collect, converge, coordinate, create, discuss, distribute, evaluate, identify, inquire, network, prepare, request and review (Hädrich 2008). However, there is a set of distinguished actions which describe the core of the step, i.e. the main activities that persons engage in when pursuing a certain step.

- Informing practices: Finally, the steps can be characterized with the help of the primarily targeted informing practice: expressing, monitoring, translating, networking (Schultze 2000) and personalization, i.e. marking a knowledge element as one's own so that one can trace back future developments towards an individual's creation in order to have the individual benefit from it.

- Knowledge elements: Organizations typically handle large number of knowledge elements for a variety of reasons. Some of them can be classified into types of knowledge elements that are typical for individual steps of the knowledge maturing process.

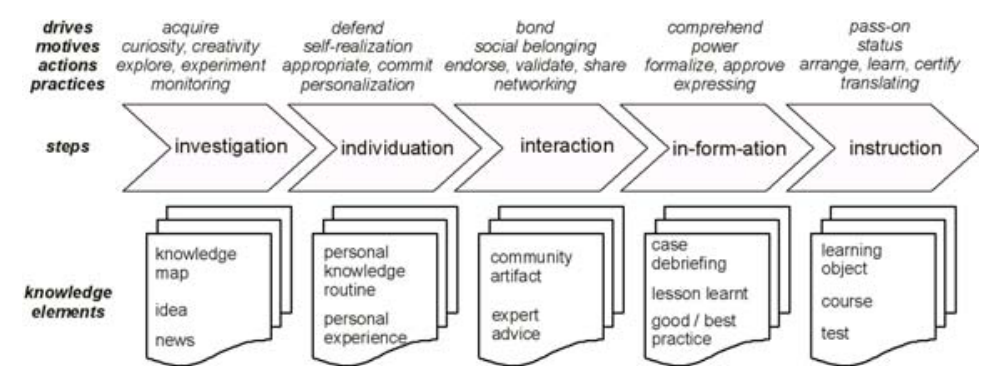

Fig. 1. Knowledge maturing model (based on Maier \& Schmidt 2007) 
The individual phases are described in the following points.

- Investigation: New ideas are developed by individuals either in highly informal discussions or by browsing the knowledge spaces available inside the organization and, with respect to SMEs, particularly beyond- e.g. in the Web. This step is driven by curiosity and creativity. The knowledge is subjective and deeply embedded in the context of the originator. The vocabulary used for communication is vague and often restricted to the person expressing the idea.

- Individuation: New ideas or results found in the investigation phase that have been enriched, refined or otherwise contextualized with respect to their use are now appropriated by the individual. This means that the individual marks his contributions so that he can benefit from its future (re-)use. The experience is thus personalized.

- Interaction: This step is driven by social motives and the benefits that individuals typically attribute to sharing knowledge. These are, among others, belonging to a preferred social group, thus increasing probability of getting back knowledge from the community when one needs it. From the perspective of semantics, this accomplishes an important maturing step, i.e. the development of common terminology shared among community members, e.g., in discussion forum entries or Blog postings.

- In-form-ation: Artifacts created in the preceding two steps are often inherently unstructured and still highly subjective and embedded in the context of the community. In this phase, purpose-driven structured documents are created, e.g., project reports or design documents or with a stronger knowledge connotation, rich case descriptions, lessons learnt or good practices, in which knowledge is desubjectified and the context is made explicit.

- Instruction: Documents produced in the preceding step are typically not well suited as learning materials because no didactical considerations were taken into account. Now the topic is refined to improve comprehensibility in order to ease its consumption or re-use. The material is ideally prepared in a pedagogically sound way, enabling broader dissemination. Individual learning objects are arranged to cover a broader subject area and thus are composed into courses. Tests and certificates confirm that participants of formal trainings have achieved a certain degree of proficiency.

Knowledge thus can be classified according to its level of maturity. The class then suggests the appropriate form of learning and technical support. The following criteria have been identified as useful to define classes of knowledge:

- Validity: Certainly, the most obvious categorization refers to a validation process and could distinguish in as a first step between unproven and proven knowledge. In a more refined version that considers the specifics of organizational knowledge, validation could take into account the number of successful uses of knowledge, systematic tests or, (mathematical) proves.

- Hardness: In analogy to mineralogy, this criterion describes the (alleged) reliability of information or knowledge. According to Watson (2005), a possible scale runs from unidentified sources of rumors up to stock exchange data.

- Context: With deepened understanding, connections to other topics become visible which play an important role in learning (Siemens 2005). This must not be confused with inherent contextualization of knowledge which decreases in the knowledge 
maturing process and refers to the degree of implicit linkage to the creation context, so that it cannot be used outside the original context. Inherent contextualization and inter-connectedness are inverse properties.

- Commitment/legitimation: Knowledge can be structured according to the amount of support it gets. Support can be in the form of commitment by members of groups, teams or communities within SMEs or in their business environment. Another form of support can be authorization to use knowledge by supervisors, executives or committees as well as legalization and standardization, i.e. forms of legitimation.

- Form of learning: As knowledge maturing is basically interconnecting individual learning processes in which knowledge is taught and learnt, an important criterion is teachability. Whereas immature knowledge is hard to teach, even to experts, formal training by definition allows for wide-range dissemination.

\section{Knowledge Maturing Services}

Knowledge maturing is used as concept to structure the core knowledge maturing services in an enterprise knowledge infrastructure that helps SMEs to streamline the IT services offered by internal or external IT service providers. This layer of core services builds upon infrastructure and integration services. The Intranet infrastructure provides basic functionality for storage, processing, synchronous and asynchronous communication, sharing of data and documents as well as management of electronic assets in general and of Web content in particular. Sources for structured and semistructured data can be classified into organization-internal and organization-external sources. In case of SMEs, organization-external sources play a particularly important role due to the fact that typically a large portion of required knowledge cannot be built and maintained without customer, supplier and partner organizations. An ontology helps to meaningfully organize and link knowledge elements that come from a variety of sources and are used to analyze the semantics of the organizational knowledge base. Integration services are needed to manage meta-data about knowledge elements and the users that work within the system. Synchronization services export a portion of the knowledge workspace for work offline and (re-)integrate the results of work on knowledge elements that has been done offline. In case of SMEs, tagging and lightweight ontologies that are developed collaboratively, such as folksonomies, are seen as a promising solution for the challenge of integrating knowledge elements from diverse sources (e.g., Braun et al. 2008, Zacharias et al. 2009).

In the following section, services to support the steps of the knowledge maturing model are briefly described.

\subsection{Investigation}

Investigation services help to identify relevant knowledge in various forms and formats, particularly documented knowledge represented by various types of information resources. This relates to information retrieval, business intelligence, data mining and visualization for exploring structured data, enhanced visualization techniques for browsing knowledge resources and functions for monitoring knowledge sources. Figure 2 presents four groups of investigation services structured by means of a cycle. 


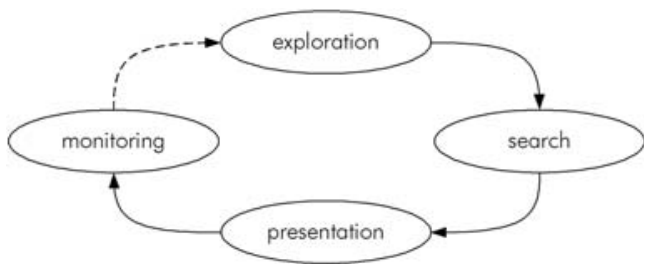

Fig. 2. Investigation services

The first two types of services are based on a distinction between two general search modes. Exploration supports a process of retrieving information whose main objectives are not clearly defined in the beginning and whose purpose might change during the interaction with a system, i.e. if users lack knowledge about the domain or if they cannot express it precisely. Important ways of support are knowledge visualizations, e.g., representations of sources of knowledge as well as their relationships. Search in contrast relates to a focused mode where a user needs to be able to describe a problem at least to some degree, e.g., by formulating a search query consisting of a number of keywords. Presentation deals with representing search results which particularly concerns ranking, visualization of relevant information as well as the obtainment of user feedback. Monitoring services support continuous non-focused scanning of the environment and gathering of useful "just in case" information.

The general sequence of these services as shown in the figure indicates that an explorative mode of search can be seen as the first step for investigating information resources. The more knowledge is acquired about a topic, the more specific becomes the information need and the required results can be formulated. Consequently, exploration is followed by a more focused search as well as by presentation of potentially relevant results. Monitoring is positioned as the last step within the cycle as it is concerned with relatively specific topics whose development is observed over different resources. The dashed line between monitoring and exploration means that monitoring may trigger further investigation cycles when it yields new fields of knowledge that should be investigated. However, the sequence of services shown in the figure only should be taken as a general ordering. Principally, investigation services can be accessed in any order. The same is true for the cycles described in the next sections.

\subsection{Individuation}

The concept of individuation so far has been neglected in many initiatives to create knowledge infrastructures. This means that many services in this category are quite rudimentary, because the other services have been deployed systematically for a much longer period of time. Many efforts have focused on transparency of knowledge and on supporting knowledge workers in sharing knowledge or even detaching knowledge from humans as "media" of knowledge. However, at least in a more individualistic culture stressing diversity, the individual knowledge worker also requires support concerning appropriation of knowledge.

Figure 3 gives an overview of four classes of services helpful for individuation including (1) reflection of individual experiences, (2) expression of ideas and proposals 
in a way that makes sense for the individual, (3) building competence and managing one's individual career and (4) achieving the final step of individual professional development, i.e. expert status in a certain knowledge domain.

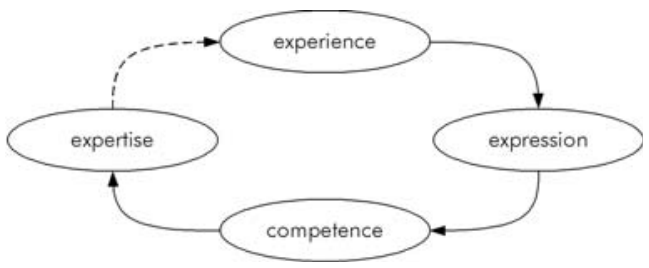

Fig. 3. Individuation services

The concept of individuation is widely used in a number of scientific disciplines, most notably psychology and philosophy. Its origin can be traced back to the Latin adjective "individuus" which means indivisible, inseparable or undivided. It describes processes in which the undifferentiated becomes individual, or processes in which separable components become an indivisible whole. When translating this rather abstract definition to knowledge infrastructures, it comprises four steps in the maturing of knowledge that can be supported by services.

- Experience: First, knowledge workers have to make sense of a vast and chaotic amount of material, e.g., the material available on a company Intranet or the Web. Thus, they shift their focus or awareness to knowledge elements which they differentiate out of the mass of material and connect to them. This might mean reading and understanding a resource on the Web and then tagging or bookmarking it in order to connect to it.

- Expression: Second, the knowledge worker then needs to make sense out of the separate knowledge elements that she has connected to in order to learn and, in subsequent steps, to build competence and expertise which means the encapsulation of knowledge elements into an inadvisable whole. This might mean connecting contents from diverse sources in order to express what the knowledge worker knows about a certain knowledge domain. It might also mean that the knowledge worker expresses some personal idea or proposal that is at first unconnected to the rest of the accessible knowledge elements.

- Competence: Competence reflects the relationship between an individual's skills and the requirements of the work to be completed by the individual. Competence services aim at supporting individual knowledge workers to develop their own knowledge and skills in a self-guided way. This includes reflecting on and making sense out of the development of individual competencies in the pursuit of a sequence of tasks.

- Expertise: While competence reflects on the fact that an individual's skills are sufficient to complete work in a certain domain, expertise reflects on an individual knowledge worker's long-standing experience in a domain of knowledge which differentiates her perception and acting inside the domain, from non-experts. Some authors extend the hierarchical model of data, information and knowledge by competence and further on by expertise which should reflect the increasing abstraction 
from the concrete happenings, but also the increasing integration of separate individual experiences into a coherent whole - an expert's profound knowledge that marks the highest (supportable) step of individuation with respect to knowledge workers' professional development.

\subsection{Interaction Services}

Understanding group work and the design of supportive ICT tools has been researched for over two decades under the topic of computer-supported cooperative work (CSCW). It is an interdisciplinary research field and was started as an effort by technologists to learn from members of other disciplines, e.g., economists, social psychologists, organizational theorists and educators (Grudin 1994, 19f). Technical support may focus on various aspects of interaction in group work as exchange of knowledge in shared workspaces, provision of communication media, structuring cooperative work processes and guidance of decision processes.

Figure 4 shows classes of interaction services. Every communicative relationship at some point is initiated. Initiation services thus target establishing links between people. Networking services target deepening, renewal and care-taking for these links. Communication services focus on exchanging knowledge between people and offer various channels to enable or enhance this. Finally, community services target supporting groups characterized by long-term, social relationships with many rather weak links between their members. Consequently, these services form a cycle of increasingly closer forms of interaction as outlined above. Not every relationship evolves through all of these stages. The dashed line between community and initiation indicates that every community may be reenergised by developing links to new people.

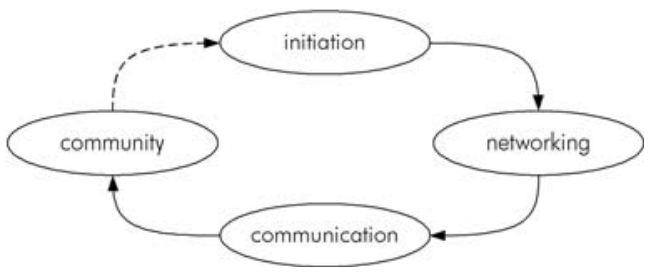

Fig. 4. Interaction services

\subsection{In-Form-Ation Services}

In-form-ation services bring knowledge into a form so that it can be easily distributed and reused. This process can be labeled as "formalization" since knowledge is expressed by means of, e.g., written language, graphical representations and formulas which involve a more or less strong formalization process in order to emphasize that individual knowledge is transformed to contextualized information instead of knowledge as soon as it is expressed and incorporated by any type of information resource. 
Figure 5 displays four general groups of in-form-ation services. Capturing refers to the process of making potentially large amounts of information resources electronically available in the required format and quality. This comprises scanning of paperbased information resources and further processes such as visual post-processing and optical character recognition. It may also include conversion and loading of legacy data in various electronic formats. Manipulation services support coordination and management of changes on information resources. Translation services concern transforming information resources into different structures and formats. They serve the important task of publishing information on the Intranet or the Web. Archiving services help managing retention periods of information resources, storing them in a secure and cost-effective manner and ultimately assist their deletion. They are relevant because legal regulations oblige organizations to keep information resources for defined periods of time and to be able to provide them as pieces of evidence.

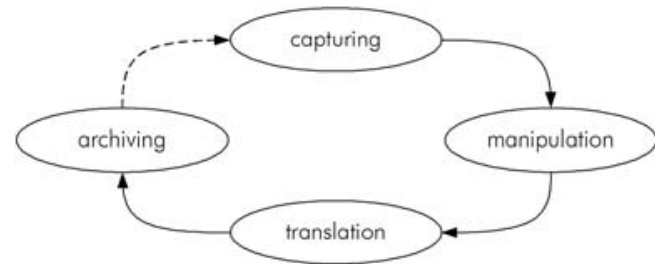

Fig. 5. In-form-ation services

\subsection{Instruction Services}

Instruction services target turning material provided in the former steps of the maturity model, particularly formal documents from the in-form-ation step, but also individual contributions from the interaction step, into resources that can be used for self-guided learning or formal instruction. Ultimate aim is to didactically refine material in order to help knowledge workers in building individual and team knowledge. Although instruction services generally can be applied for all types of knowledge, they are primarily instruments to facilitate communication and to transfer implicit, personal knowledge. Figure 6 shows four main categories used to subdivide instruction services.

Contents produced in the preceding steps of the maturing model are typically not well-suited for supporting self-guided learning or formal training. Enrichment services help knowledge workers to refine material deemed suitable for learning with the help of pedagogical and didactical approaches. Composition services use prepared learning material or learning objects as input for creating courses, i.e. arrangements of related learning material or objects. Consumption marks the transition between design time of learning resources and run-time. It primarily consists of using courses delivered to learners by a run-time environment such as a learning management system which needs to be administered. Finally, assignment services describe test and examination instruments that can be used in order to evaluate what has been learned. 


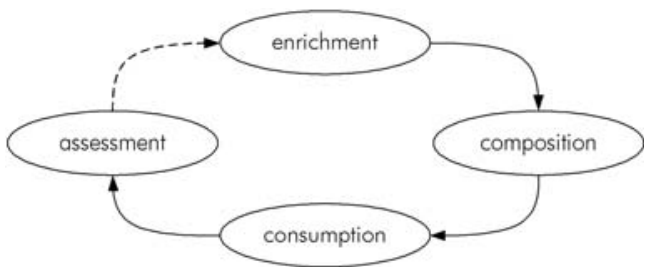

Fig. 6. Instruction services

\section{Discussion and Conclusion}

Generally, SMEs are on the one hand in a relatively good position with respect to handling of knowledge compared to large organization because of factors like a lower level of knowledge fragmentation and spreading across geographical entities, low degree of formalization and specialization, strong ties between members of the organization, direct access to "knowers", hardly restricted access to documented knowledge, stronger identification of employees with the organization and an often tighter network of informal contacts between employees. On the other hand, it is less likely to find required expertise within the organizational boundaries resulting in a higher need to cooperate, it is often difficult to recruit talent, there are no specific roles that support or guide knowledge activities and, last, but not least, there are little financial resources or time to be allocated to KM initiatives.

This results in a situation in many SMEs that introducing centralized KM systems is seen as not feasible and consequently many employees rely on their own arrangement of ICT services to support their knowledge activities. This calls for flexible ways of arranging services supporting knowledge activities when and by whom they are needed. As one cannot expect large amounts of time and resources for guidance, pragmatic models that allow for a quick analysis of the services that are currently supporting employees in their knowledge activities are needed.

This is what the knowledge maturing model and the corresponding services as described above are intended for. With their help, one could analyze local "good practices" of individual employees (investigation, individuation), informal collections of employees, also called communities (individuation, interaction), teams and work groups (interaction, in-form-ation), the formal handling of documented knowledge (in-form-ation) as well as apprenticeship, mentor-mentee and other relationships with a teaching part (instruction). Specific to SMEs could be an endeavor to extend the scope of analysis to business partners, particularly "good practices" found in supplier, customer and cooperation partner organizations due to close relationships that have to be built with them in order to foster knowledge cooperation.

Moreover, as argued in the section "knowledge services", SMEs can potentially benefit substantially from contents and services offered in a Web 2.0 manner over the Internet. Consequently, "good practices" supposedly include an increasing portion of Web 2.0 contents and services. The knowledge maturing model can be applied to govern the use of such contents and services. For example, services supporting each phase that have been initiated by individual employees can be selected to be shared, described, recommended or even composed into the SME's ICT infrastructure using 
mashup or SOA technology so that compliance can be assured with respect to the regulations applicable to the organization. A next step could be the analysis of gaps between the services applied to support the individual steps in the knowledge maturing model. Finally, arrangements of services targeting individual steps as well as the connections between them could be suggested as a flexibly configurable knowledge infrastructure.

In summary, this paper presented the knowledge maturing model together with a structured list of knowledge services and discussed their potential support from the perspective of SMEs. The paper gave several examples of how this model could be used as a pragmatic instrument to help SMEs in exploiting the promised benefits of Web 2.0 services and deploying a KM approach that can be characterized as "lightweight", "on-demand" and "just-in-time" (Davenport \& Glaser, 2002; Tsui, 2005) as opposed to the often far-reaching, resource-intensive and centralized KM approaches suggested for large organizations.

\section{Acknowledgement}

This work was partly funded by the European Commission under the Information and Communication Technologies (ICT) theme of the 7th Framework Programme (FP7) within the Integrating Project MATURE (contract no. 216356).

\section{References}

Alonso, G., Casati, F., Kuno, H., Machiraju, V.: Web services. Concepts, architectures and applications, Berlin (2004)

April, K.A.: Guidelines for developing a k-strategy. Journal of Knowledge Management 6(5), 445-456 (2002)

Alvesson, M.: Knowledge work and knowledge-intensive firms, Oxford (2004)

Braun, S., Schmidt, A., Walter, A., Zacharias, V.: The Ontology Maturing Approach to Collaborative and Work-Integrated Ontology Development: Evaluation Results and Future Directions. In: International Workshop on Emergent Semantics and Ontology Evolution (ESOE), ISWC 2007, Busan/Korea (2007)

Davenport, T.H., Glaser, J.: Just-in-time delivery comes to knowledge management. Harvard Business Review, 5-9 (July 2002)

Davenport, T.H., Jarvenpaa, S.L., Beers, M.C.: Improving knowledge work processes. Sloan Management Review 37(4), 53-65 (1996)

Drucker, P.F.: The age of social transformation. The Atlantic Monthly 274(5), 53-80 (1994)

Grudin, J.: Computer-Supported Cooperative Work: History and focus. IEEE Computer 5(27), 19-26 (1994)

Hädrich, T.: Situation-oriented Provision of Knowledge Services. PhD Thesis, Martin-LutherUniversity of Halle-Wittenberg 2008 (2008)

Hansen, M.T., Nohria, N., Tierney, T.: What's your strategy for managing knowledge. Harvard Business Review, 680-689 (March-April 1999)

Krafzig, D., Banke, K., Slama, D.: Enterprise SOA: Service-oriented architecture best practices, Upper Saddle River (2005)

Maier, R.: Knowledge management systems. Information and communication technologies for knowledge management, 3rd edn., Berlin (2007) 
Maier, R., Hädrich, T., Peinl, R.: Enterprise knowledge infrastructures, 2nd edn., Berlin (2009)

Maier, R., Schmidt, A.: Characterizing Knowledge Maturing. A Conceptual Process Model for Integrating E-Learning and Knowledge Management. In: Proceedings of WM 2007 - 4th Conference on Professional Knowledge Management. Experiences and Visions, Potsdam 2007, pp. 325-333 (2007)

Maier, R., Thalmann, S.: Institutionalised collaborative tagging as an instrument for managing maturing learning and knowledge resources. International Journal of Technology Enhanced Learning (IJTEL) 1(1), 70-84 (2008)

Maslow, A.H.: A Theory of Human Motivation. Psychological Review 50, 370-396 (1943)

Ordóñez de Pablos, P.: Knowledge management and organizational learning: Typologies of knowledge strategies in the spanish manufacturing industry from 1995 to 1999 . Journal of Knowledge Management 6(1), 52-62 (2002)

O'Reilly, T.: What Is Web 2.0. Design Patterns and Business Models for the Next Generation of Software (2005),

http://www. oreillynet.com/pub/a/oreilly/tim/news/2005/09/30/ what-is-web-20.html (30.9.2005)

Schmidt, A.: Knowledge Maturing and the Continuity of Context as a Unifying Concept for Integrating Knowledge Management and E-Learning. In: Proceedings of I-KNOW Conference 2005, Graz (2005)

Schultze, U.: A Confessional Account of an Ethnography about Knowledge Work. Management Information Systems Quarterly 1(24), 1-39 (2000)

Siemens, G.: Connectivism: Learning as Network Creation, elearnspace (2005),

http: / /www.elearnspace.org/Articles/networks.htm

(last access: 2008/12/12)

Starbuck, W.H.: Learning by Knowledge-Intensive Firms. Journal of Management Studies 29(6), 713-740 (1992)

Tsui, E.: The role of IT in KM: Where are we now and where are we heading? Journal of Knowledge Management 9(1), 3-6 (2005)

Watson, R.T.: Data Management. Data Bases \& Organizations, 5th edn., New York (2005)

Watson, R.T.: Evolutionary Information Systems. Presentation at the University of Innsbruck (2008)

W3C. Web services glossary (2004), http: / /www.w3 .org/TR/2004/NOTE-ws-gloss-20040211/ (last access: 2008/12/12)

Wolff, E.N.: The growth of information workers. Communications of the ACM 48(10), 37-42 (2005)

Zacharias, V., Braun, S., Schmidt, A.: Social Semantic Bookmarking with SOBOLEO. In: Murugesan, S. (ed.) Handbook of Research on Web 2.0, 3.0 and X.0: Technologies, Business, and Social Applications (2009)

Zack, M.H.: Developing a knowledge strategy. California Management Review 41(3), 125-145 (1999) 